\title{
Upgrade of MEG experiment
}

\author{
Yusuke Uchiyama*† \\ ICEPP, The University of Tokyo \\ E-mail: uchiyama@icepp.s.u-tokyo.ac.jp
}

We present the upgrade program of MEG experiment to search for the lepton-flavor-violating decay, $\mu^{+} \rightarrow \mathrm{e}^{+} \gamma$, aiming for a sensitivity enhancement of one order of magnitude compared to present MEG. The key feature of this program is to increase the rate capability of all detectors to enable running at more than twice higher beam intensity, which is the intensity frontier of DC muon beam provided by PSI accelerator facility, with significantly improving the efficiency and resolutions of detectors at both positron and gamma sides. Data-taking could start in 2016 and the goal sensitivity will be achieved for a running time of 3 years. The upgraded MEG will push the unprecedented exploration of physics beyond the SM and lead the intensity frontier of particle physics in the LHC era.

The European Physical Society Conference on High Energy Physics -EPS-HEP2013

18-24 July 2013

Stockholm, Sweden

\footnotetext{
* Speaker.

${ }^{\dagger}$ On behalf of the MEG collaboration.
} 


\section{Introduction}

The conservation of lepton flavor in the standard model (SM) is considered to be accidental; there is no explicit gauge symmetry for it. Even in the minimum extension of SM with finite but tiny neutrino masses, lepton-flavor violating processes in charged lepton sector (cLFV) are highly suppressed and out of experimental reach $\left(\mathscr{B}<10^{-54}\right)$. However, introduction of new physics generally induces cLFV, possibly as large as the existing experimental limits. An observation of cLFV process can be a clear indication of new physics beyond the SM.

We have carried out the MEG experiment at Paul Scherrer Institut (PSI) to search for the $\mu^{+} \rightarrow \mathrm{e}^{+} \gamma$ decay since 2008. We established the most stringent limit on the existence of the decay; $\mathscr{B}\left(\mu^{+} \rightarrow \mathrm{e}^{+} \gamma\right)<5.7 \times 10^{-13}$ at $90 \%$ C.L. using data collected in 2009-2011 [1, 2]. The data acquisition was completed at the end of August 2013, and now we are analyzing the full data towards the final result of MEG. In parallel, we are preparing upgrading the experiment to push the search with another order of magnitude better sensitivity. In this paper, we describe the program.

\section{Motivations and goal}

Against many expectations of the discovery of $\mu^{+} \rightarrow \mathrm{e}^{+} \gamma$ decay in the range of the branching ratio $10^{-12}-10^{-13}$ from well motivated theoretical predictions, we have not yet find the decay. Even we will continue the search in the middle of $10^{-13}$ by doubling the data statistics of MEG, further exploration in $\mathrm{O}\left(10^{-14}\right)$ is highly scientifically motivated. In particular, the absence of new physics in the intensive searches in the energy frontier with LHC pushes the importance of indirect searches, especially in the lepton sector.

Improvement of the sensitivity of MEG as the increase of data statistics now starts to slow down because of the finite background. At the same time, our deep understanding of the experiment and recent technical developments enable us to build better experimental apparatus by upgrading present system. By exploiting currently available highest intensity beam, it is found we can enter the search in $\mathrm{O}\left(10^{-14}\right)$ sensitivity if we can successfully upgrade the detectors. Thus, we set our goal of the upgrade project to sensitivity reach to $5 \times 10^{-14}$, ten times better than the present MEG goal. Considering other projects in cLFV searches, this sensitivity goal is well motivated. One of our advantages is we can accomplish the project in a short time scale with a lower cost by exploiting the present apparatus and beam-line. We should carry out the program in time scale of 5 years, earlier than the next generation cLFV searches.

\section{Upgrade project}

To achieve the goal, first of all we need $\sim 10$ times larger data statistics. We realize this in the following ways; 2-3 times higher beam intensity, twice higher efficiency and an efficient usage of the beam time with stabler detectors. Then, we have to significantly suppress the background, especially the accidental background whose rate is proportional to the square of the instantaneous beam intensity. This is accomplished mainly by improving detector resolutions but by additional ways of background reduction as well. Figure 1 shows an overview of the upgrade project as comparisons with present experiment. Here, we describe each element of it. Each of them is indispensable for the goal. 


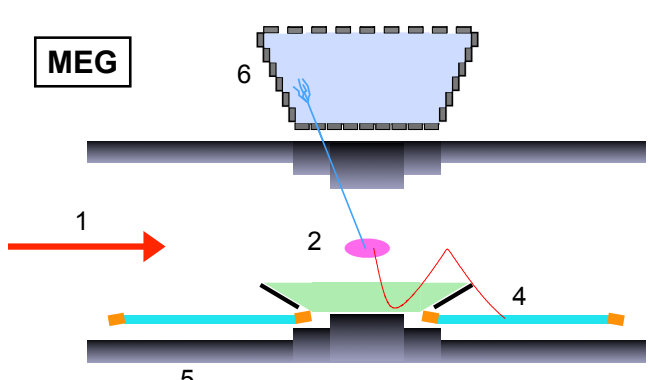

5

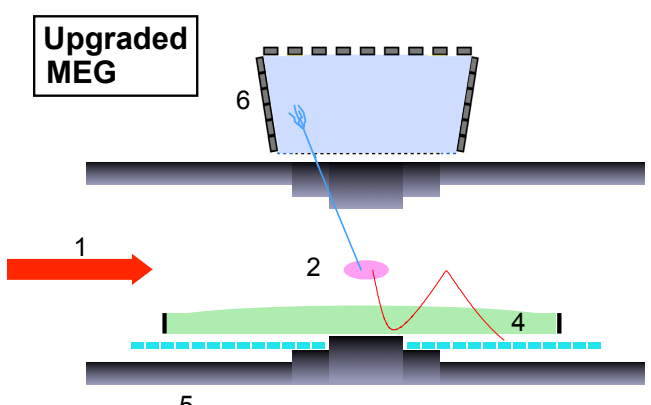

5
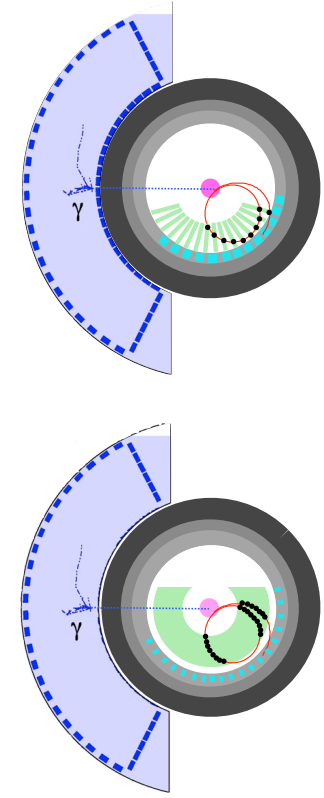

Figure 1: Overview of the MEG upgrade.

\subsection{Beam and target}

It is already demonstrated that $\pi \mathrm{E} 5$ beam-line at PSI, where we have operated MEG, can provide $\sim 3$ times higher intensity of surface muons. The efficiency of stopping muons in the target is slightly decreased because of the higher momentum spread for higher intensity, and it depends on the thickness of the target. The thickness of the target has impacts on the following points as well: a degradation of positron measurement due to the multiple-Coulomb-scattering inside the target and the generation of undesired photons originating from the annihilation-in-flight of positrons (AIF). The optimization of sensitivity comes to the baseline design; a thinner target of $140 \mu \mathrm{m}$ (from $205 \mu \mathrm{m}$ in the present configuration) thickness placed with a slant angle of $15^{\circ}$ (from $20^{\circ}$ ), and a muon stopping rate of $7 \times 10^{7} / \mathrm{sec}$ (from $3 \times 10^{7} / \mathrm{sec}$ ).

An alternative option is under consideration for the target where we use scintillating fibers as the target (active target) to measure the muon decay vertex in vertical coordinate. This additional measurement will result in a higher momentum resolution. On the other hand, the multiplescattering effect and AIF photon generation will be increased by the thicker target. The feasibility of detecting emitted positrons with a good efficiency is now under investigation using prototypes.

\subsection{Positron spectrometer}

The basic idea of positron spectrometer will be kept; an efficient measurement of high rate positrons with a special gradient magnetic field. Thus, the superconducting magnet will be reused. The components of the sub-detectors put inside the magnet will be renewed. The new spectrometer is schematically shown in Fig. 2. 


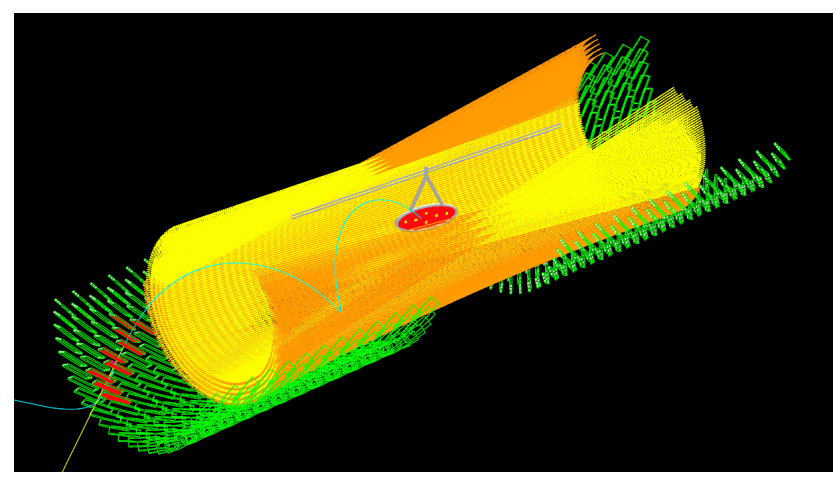

Figure 2: Schematic of the new positron spectrometer.

The present tracking detector, a set of modularized drift chambers (DC), will be replaced with a longer single-volume stereo-wire drift chamber. We can recover the loss in positron detection efficiency with the long configuration; about half of positrons at signal energy (52.8 MeV) are lost in current configuration due to hits on the support frame of DC and so forth, while in the new configuration, material on the way of the positrons is minimized. The detection efficiency is expected to be doubled. The length of the chamber would be $\sim 190 \mathrm{~cm}$. The number of sense wires is about 12000 , and the cell size is about $7 \mathrm{~mm}$ square. The chamber gas will be helium and isobutane (about 90:10). Only the light gas and wires are there in the tracking volume; in total about $1.7 \times 10^{-3} X_{0}$ per positron trajectory. The effect of multiple scattering on the tracking is minimized and the generation of photons through AIF is also reduced from the current system. The tracking resolutions will be improved mainly by the increase in the number of hits. The drift-distance resolution is expected to be $\sim 130 \mu \mathrm{m}$, and with a small prototype detector we have achieved $\sim 100 \mu \mathrm{m}$ resolution for the gas configuration. The longitudinal hit position is reconstructed by the charge division between the two wire ends, the time difference and the stereo angle of wires. The redundant measurements enable pattern recognitions of the tracks at the high rate. Another important requirement for the system is stability in both instantaneous operation at high rate and long-term operation. We are now performing aging tests with a high intensity $\mathrm{X}$-ray source to accelerate the effect. We have tested up to $0.5 \mathrm{C} / \mathrm{cm}$ which is about twice exposure of the innermost wire without observing severe deterioration in the performance.

The timing of positron is measured by two sets of timing counters (TC) after exiting DC. In the present experiment, we use arrays of long and thick scintillator bars $\left(800 \times 40 \times 40 \mathrm{~mm}^{3}\right)$ readout by PMTs at the both ends. These counters will be replaced with pixelated counter arrays consisting of smaller and thinner scintillator plates readout by SiPMs [3]. The new TC is designed for high momentum positron to hit several counters. We can significantly improve the time resolution by the multi-counter measurements. This works not only for improvement in the intrinsic counter resolution but also for reduction of electronics and calibration contributions. The single counter performance has been investigated and optimized through tests with ${ }^{90} \mathrm{Sr} \beta$-source. We adopt a ultra-fast scintillator (BC-422) and six SiPMs (three at each end connected in series). The dimension of a counter was optimized by considering the trade-off effect between the single counter resolution (the smaller, the better) and the hit multiplicity for a positron measurement (the 
larger, the better). For the current baseline design $\left(90 \times 40 \times 4 \mathrm{~mm}^{3}\right)$, the single counter resolution is $\sim 65$ psec and the average multiplicity is $\sim 7$ counters for a signal positron. This results in an overall time resolution of 30-35 psec better than the half of present resolution. The principle of the multi-counter scheme and the overall resolution were demonstrated by a beam test.

Another improvement in the positron time measurement is foreseen from the improved reconstruction of the trajectory from the target to TC. Because we can track the path up to the entrance to TC owing to the longer DC on contrast to a large gap between DC and TC in current configuration, the accuracy of path-length, hence the time-of-flight to be subtracted from the TC hit time will be improved to be negligible $(<10 \mathrm{psec})$ from one of the major contributions ( $\sim 70 \mathrm{psec})$ to the total time resolution.

\subsection{Photon detector}

The innovative photon detector consisting of liquid xenon (LXe) as a scintillation media shows high performance in energy, timing and position measurements of the incident photon. However, it shows a few weak points, especially at the edge of its acceptance. To overcome the weak points, we will upgrade the LXe photon detector in two-fold ways as shown in Fig. 3. One is a re-arrangement of lateral wall PMTs to reduce the shower escape from those faces and to improve the light collection uniformity when the photon conversion occurs close to the lateral wall PMTs.

The other, major modification is a replacement of the 2-inch PMTs on the photon-incident wall with smaller photo-sensors, SiPMs. This is for overcoming the poor resolution for shallow events-events when the conversions of incident photons occur close to the incident wall. With large PMTs, the collection efficiency of scintillation light heavily depends on the relative position of the conversion to the PMT positions. This can be overcome with smaller sensors put densely on the incident wall to improve the uniformity of the light collection. We have been developing a new MPPC (a type of SiPM from Hamamatsu Photonics) which has sensitivity to UV light around $175 \mathrm{~nm}$ wavelength. We have already achieved a photo-detection-efficiency of $\sim 17 \%$ for xenon scintillation light which are comparable with MEG PMT [4, 5]. The sensor size is another important parameter to suppress the number of readout channels to a realistic number. We have successfully developed $12 \times 12 \mathrm{~mm}^{2}$ size MPPC with a monolithic array-large enough for our application. With this size sensors, one PMT will be replaced by 16 MPPCs, and in total about 4000 MPPCs will be placed on the incident face. With these increased number of 'eyes', the imaging power for the shower will be significantly improved as you can imagine from Fig. 4. From a detail simulation study, the energy resolution as well as position resolution for shallow events are found to be improved significantly [4]. The detection efficiency of signal photons will also be improved by $\sim 10 \%$ because of the thinness of MPPC compared to the PMT.

\subsection{Electronics}

To cope with the increased number of readout channels, the trigger and readout electronics will be also upgraded. A new digitization board is being developed. The trigger and readout electronics are integrated on a same board in a compact-dense custom crate. The high-speed waveform digitizing chip, DRS4 [6] same one used in current MEG will be mounted for the readout but the bandwidth will be improved to $>700 \mathrm{MHz}$. The new board integrates switchable amplifiers as well as high voltage supplies for SiPMs. 


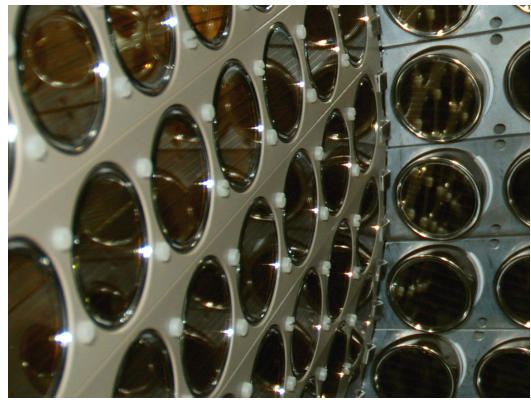

(a) Present

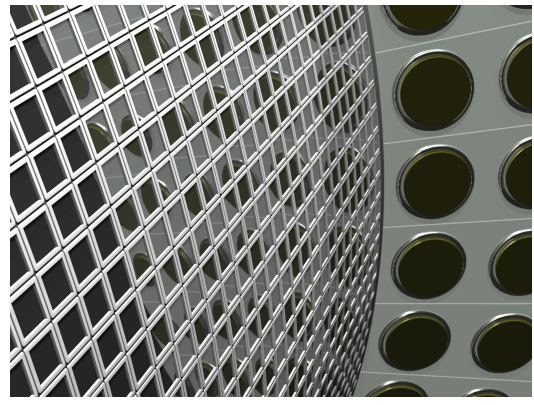

(b) Upgraded

Figure 3: (a) Picture of PMT arrangement in current photon detector. (b) Computer graphics of upgraded detector with SiPMs on the incident face and rearranged PMTs on the lateral faces.

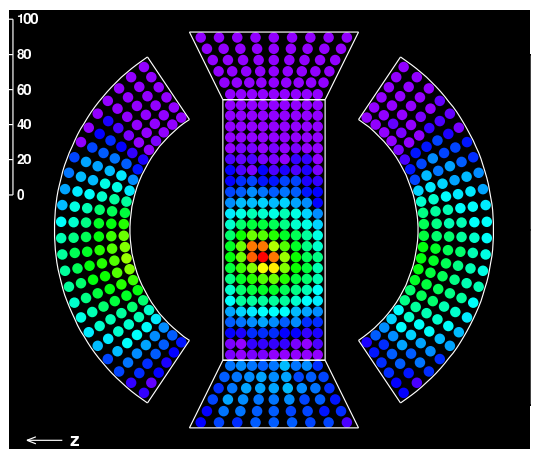

(a) Present

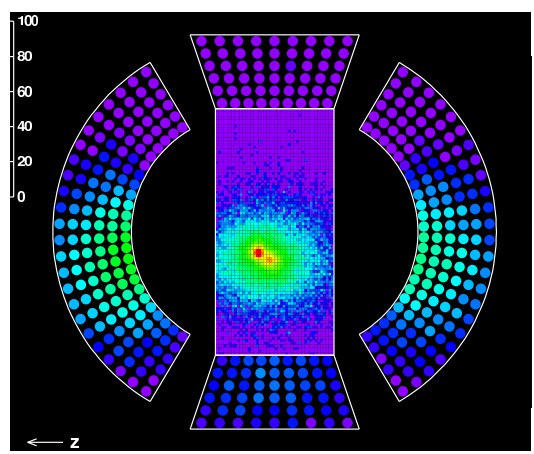

(b) Upgraded

Figure 4: Views of a simulated event by present (a) and upgraded (b) photon detector. The color shows the amount of charge density collected by corresponding photo sensor. Two photons ( 28 and $25 \mathrm{MeV}$ ) enter in this example (pile-up event).

\subsection{Further background reduction}

We can suppress the background not only by the improvement in detector resolutions but in other ways. Suppressing photon generation by reducing material in the target and tracking volume is one way. The photon yield from AIF producing energy deposition in LXe larger than $48 \mathrm{MeV}$ will be reduced by $35 \%$ in the upgraded configuration. Furthermore, AIF origin background can be identified in offline analysis when the AIF occurred in the tracking volume by tagging the corresponding positron trajectory left in the tracker before the AIF process. The analysis is now in progress for present MEG data and the impact seems promising. Note that the tagging power will be increased by the continuously measured tracks by the single-volume DC.

Another main source of high-energy photons is the radiative muon decay (RMD). In the current configuration, half of photons $>48 \mathrm{MeV}$ come from RMD and the other half from AIF. Photons from RMD can be identified by tagging the low-momentum positron emitted in the process. To detect the positrons, we are developing an additional optional detector placed on the beam axis. From a simulation study, the tagging efficiency is expected to be $\sim 70 \%$ for RMD events with photon energy $>48 \mathrm{MeV}$, resulting in the sensitivity enhancement of $10-15 \%$. The principle of the new idea as well as the stable operation of the detector in a high-rate environment are tested in 
Table 1: Summary of the performance.

\begin{tabular}{lll}
\hline Resolution $(\sigma)$ & Present & Upgrade \\
\hline $\mathrm{e}^{+}$energy $(\mathrm{keV})$ & 306 & 130 \\
$\mathrm{e}^{+} \theta(\mathrm{mrad})$ & 9.4 & 5.3 \\
$\mathrm{e}^{+} \phi(\mathrm{mrad})$ & 8.7 & 3.7 \\
$\mathrm{e}^{+}$vertex $(\mathrm{mm})$ & $2.4 / 1.2$ & $1.6 / 0.7$ \\
$\gamma$ energy $(\%)$ & $2.4 / 1.7$ & $1.1 / 1.0$ \\
$\gamma$ position $(\mathrm{mm})$ & $5 / 5 / 6$ & $2.6 / 2.2 / 5$ \\
$\gamma$ - $\mathrm{e}^{+}$timing $(\mathrm{ps})$ & 122 & 84 \\
\hline Efficiency $(\%)$ & & \\
\hline trigger & $\approx 99$ & $\approx 99$ \\
$\gamma$ & 63 & 69 \\
$\mathrm{e}^{+}$ & 40 & 88 \\
\hline
\end{tabular}

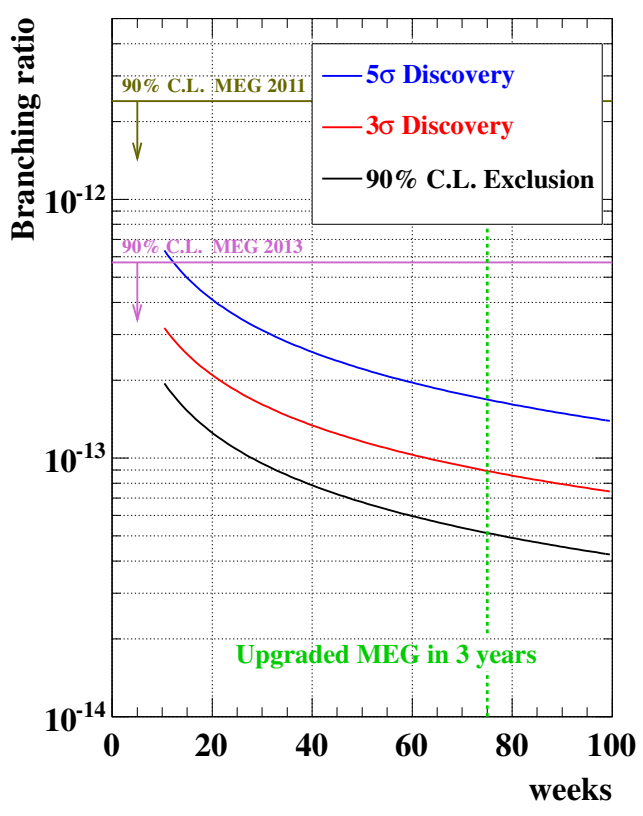

Figure 5: Sensitivity curves.

beam tests.

\section{Prospects}

Table 1 summarizes the detector performance. The expected sensitivity is calculated by using the same analysis framework as presently used with the expected detector performance and the higher accidental background rate due to the higher beam intensity. Figure 5 shows the upper-limit and discovery sensitivities as a function of DAQ time for the baseline configuration. The expected upper limit on the $\mu^{+} \rightarrow \mathrm{e}^{+} \gamma$ branching ratio assuming null-hypothesis reaches $5 \times 10^{-14}$ at $90 \%$ confidence level after three years DAQ.

The proposal of MEG upgrade [7] was approved by the PSI committee in January 2013. We plan to start physics run in 2016 through a couple of years R\&D and commissioning from now. The upgraded MEG will keep leading the intensity frontier of particle physics for more than next 5 years and push the unprecedented exploration of physics beyond the SM.

\section{References}

[1] J. Adam et al. [MEG collaboration], Phys. Rev. Lett. 110 (2013) 2018012.

[2] F. Cei, in these proceedings.

[3] W. Ootani, Nucl. Instrum. Methods Phys. Res., Sect. A, doi:10.1016/j.nima.2013.07.043 (in press).

[4] D. Kaneko, Nucl. Instrum. Methods Phys. Res., Sect. A, doi:10.1016/j.nima.2013.06.008 (in press).

[5] K. Sato et al., Nucl. Instrum. Methods Phys. Res., Sect. A, doi:10.1016/j.nima.2013.06.054 (in press).

[6] S. Ritt, R. Dinapoli, U. Hartmann, Nucl. Instrum. Methods Phys. Res., Sect. A 623 (2013) 486-488.

[7] A.M. Baldini et al., arXiv:1301.7225 [physics.ins-det]. 